\title{
The influence of mutual perturbations on the eccentricity excitation by jet acceleration in extrasolar planetary systems
}

\author{
F. Namouni · J. L. Zhou
}

Received: 24 November 2005/Accepted: 13 March 2006/

Published online: 15 August 2006

(C) Springer Science + Business Media B.V. 2006

\begin{abstract}
We study the influence of mutual planetary perturbations on the process of eccentricity excitation by jet acceleration suggested by Namouni (Astron. J. 130, 280-294). Modeling the jet's action by a constant-direction acceleration, we solve the linear secular equations of the combined planetary perturbations and the jet acceleration of the host star for a twoplanet system. The effects of the acceleration's strength, relative mass ratio and the relative distance of the two planets are investigated. The model is applied to the extrasolar planetary systems of HD 108874, 47 Uma, and HD 12661.
\end{abstract}

Keywords Extrasolar planets $\cdot$ Stellar jets $\cdot$ Eccentricity excitation

\section{Introduction}

Ten years after the discovery of the first Jupiter-like planet around 51 Pegasi, the number of extrasolar planets is steadily on the rise. As of this writing, we know of 173 planets and 18 multiplanet systems (see the extrasolar planetary encyclopaedia at http://www. obspm.fr/planets). Half of these planets have orbital eccentricities in excess of 0.28 . Such large eccentricities are surprising as it is thought that planets form in a protoplanetary cloud on nearly circular orbits much like the planets of the solar system. In effect, these observations seem to have moved the paradigm of planetary formation away from the solar system. Possible explanations for the large eccentricities include the perturbations that follow planet-planet scattering (Marzari and Weidenschilling 2002; Ford et al. 2003), the tidal

F. Namouni $(\bowtie)$

CNRS, Observatoire de la Côte d'Azur, BP 4229, 06304 Nice, France

email: namouni@obs-nice.fr

\section{J. L. Zhou}

Department of Astronomy, Nanjing University, Nanjing 210093, China

e-mail: zhoulj@nju.edu.cn 
interaction between the gas disk and the planets (Goldreich and Tremaine 1981), Kozai's secular eccentricity cycles (Holman et al. 1997; Terquem and Papaloizou 2002; Takeda and Rasio 2005), the eccentricity's pumping during planetary pair migration in mean motion resonance (Chiang et al. 2002; Lee and Peale 2002), the perturbations by stellar encounters (Zakamska and Tremaine 2004) and the outcome of a stellar-like relaxation that occurred if planets formed by gravitational instability (Papaloizou and Terquem 2001). The merits and drawbacks of most of these mechanisms are well documented (Tremaine and Zakamska 2004). A main recurring criticism is that when these mechanisms work they do so for specific systems. This lack of generality suggests that in reality various combinations of these mechanisms are at work in all systems. While satisfactory, such a conclusion does not explain (1) why the solar system is such an outlier, (2) what gives rise to apsidal alignment in nonresonant systems, and (3) what the origin of the similarity of the eccentricity distributions of extrasolar planets and spectroscopic binary stars is (Stepinski and Black 2001).

Looking for possible mechanisms that can explain the states of both solar and extrasolar planets, Namouni (2005) (hereafter N05) reasoned that the similarity in the eccentricity distribution of extrasolar planets and spectroscopic binary stars may be a hint at a common excitation mechanism. If this were true then such a mechanism must depend weakly on the local dynamics of the companion. The description of the corresponding physical process would therefore, depend weakly on the companion's position and velocity. The simplest possible process satisfying this condition is a constant acceleration. To complete the description of the physical process, the direction of its acceleration too has to be independent of the planetary plane. A possible choice is the star's rotation axis, which naturally hints at stellar jets and star-disk mass loss as natural mechanisms for such an acceleration.

Typical stellar jet accelerations of magnitudes $A \sim 10^{-11}$ to $10^{-13} \mathrm{~km} \mathrm{~s}^{-2}$ are capable of exciting planetary eccentricities. The acceleration's parameters are best known for multiple systems because mutual gravitational perturbations cause the planetary orbits to precess. If the secular frequencies associated with these precessions are larger than the jet acceleration's excitation frequency, the efficiency of eccentricity excitation is hampered. To verify the validity of the eccentricity excitation by jet acceleration, it is necessary to quantify its relative importance with respect to planetary perturbations in specific multiple systems. Such an approach has been applied in N05 to the Jupiter-Saturn and $v$ Andromedae binary system using direct numerical integration of the equations of motion. It was shown that the excitation by jet acceleration was able to explain the observed states of these systems.

In this paper, we develop a fast semi-analytic approach to study the effect of mutual gravitational perturbations in a two-planet system whose host star is subject to an acceleration by a material jet. We assume that the jet's intrinsic precession frequency is small compared to its excitation frequency $n_{\mathrm{A}}=3|A| / n a$ where $n$ and $a$ are the mean motion and semi-major axis of the planet, respectively. This puts the jet resonance studied in N05 much closer to the star than the location of the planetary region allowing us to ignore the complex issues of eccentricity damping interior to the resonance and resonant eccentricity driving. We also assume that the two-planets are not in mean motion resonance.

The paper is organized as follows: in Sect. 2, we lay down the analytical model based on secular perturbation theory applied to a system consisting of two planets and a star accelerated by a jet. In Sect. 3, we compare the secular semi-analytical model to the direct integration of the equations of motion. The effects of the acceleration's strength, the planetary mass ratio, and relative distance are discussed in Sects. 4-6, respectively. The secular model is applied to the planets of HD 108874, 47 Uma, and HD 12661 in Sect. 7, where we derive the amplitudes of the secular eigenmodes and determine the minimum acceleration strength required for efficient excitation by a slowly-precessing acceleration. Section 8 contains a summary. 


\section{Linear perturbations}

We consider the system made up of two planets orbiting a host star that is accelerated by an asymmetric jet. It has been shown in N05 that the jet's acceleration perturbs a planetary orbit through the potential:

$$
\langle R\rangle_{\text {jet }}=-\frac{3}{2} a \mathbf{A} \cdot \mathbf{e}=-\frac{3}{2} A a e \sin (\varpi-\Omega) \sin I,
$$

where $\mathbf{e}=\mathbf{v} \times \mathbf{h} / G(m+M)-\mathbf{x} /|\mathbf{x}|$ is the eccentricity vector of magnitude $e$, which is a constant of the Keplerian motion and can be expressed in terms of the position $\mathbf{x}$, the velocity $\mathbf{v}$, the angular momentum $\mathbf{h}$, the gravitational constant $G$, and the masses of the star $M$ and that of its planetary companion $m$. The potential also depends on the orbital semi-major axis of the planet, $a$. The last expression is obtained by choosing the $z$-axis along $\mathbf{A}$; in this case, $\varpi, \Omega$, and $I$ are the longitude of pericenter, the longitude of ascending node, and the inclination of the orbit with respect to $\mathbf{A}$.

The two planets are assumed not to be in mean motion resonance. This allows us to use the classical secular perturbation potential of first-order in the masses and second-order in eccentricity and inclination to describe the long-term motion of the two planets. Its expression is given as (see, e.g. Murray and Dermott 1999):

$$
\begin{aligned}
\langle R\rangle_{\text {planets }}= & \frac{1}{8}\left[2 \alpha \partial+\alpha^{2} \partial^{2}\right] b_{1 / 2}^{(0)}\left(e_{1}^{2}+e_{2}^{2}\right)-\frac{1}{8} b_{3 / 2}^{(1)}\left(I_{1}^{2}+I_{2}^{2}\right) \\
& +\frac{1}{4}\left[2-2 \alpha \partial-\alpha^{2} \partial^{2}\right] b_{1 / 2}^{(1)} e_{1} e_{2} \cos \left(\varpi_{1}-\varpi_{2}\right) \\
& +\frac{1}{4} \alpha b_{3 / 2}^{(1)} I_{1} I_{2} \cos \left(\Omega_{1}-\Omega_{2}\right)
\end{aligned}
$$

where $\alpha$ is the ratio of the inner planet's semi-major axis to that of the outer planet $a_{1} / a_{2}$, $\partial$ is the partial derivative with respect to $\alpha$, and $b_{1 / 2}^{(0)}, b_{1 / 2}^{(1)}$ and $b_{3 / 2}^{(1)}$ are the usual Laplace coefficients.

The evolution equations for the eccentricity and inclination of the two planets are obtained from the Laplace-Lagrange equations for the the eccentricity and inclination vectors $\left(h_{i}=\right.$ $e_{i} \sin \varpi_{i}, k_{i}=e_{i} \cos \varpi_{i}, p_{i}=I_{i} \sin \Omega_{i}, q_{i}=I_{i} \cos \Omega_{i}, i=1$ or 2) as:

$$
\begin{aligned}
& \dot{h}_{i}=\frac{1}{n_{i} a_{i}^{2}} \frac{\partial}{\partial k_{i}}\left[\frac{G m_{j}}{a_{2}}\langle R\rangle_{\text {planets }}+\langle R\rangle_{\text {jet }}^{(i)}\right], \\
& \dot{k}_{i}=-\frac{1}{n_{i} a_{i}^{2}} \frac{\partial}{\partial h_{i}}\left[\frac{G m_{j}}{a_{2}}\langle R\rangle_{\text {planets }}+\langle R\rangle_{\text {jet }}^{(i)}\right], \\
& \dot{p}_{i}=\frac{1}{n_{i} a_{i}^{2}} \frac{\partial}{\partial q_{i}}\left[\frac{G m_{j}}{a_{2}}\langle R\rangle_{\text {planets }}+\langle R\rangle_{\text {jet }}^{(i)}\right], \\
& \dot{q}_{i}=-\frac{1}{n_{i} a_{i}^{2}} \frac{\partial}{\partial p_{i}}\left[\frac{G m_{j}}{a_{2}}\langle R\rangle_{\text {planets }}+\langle R\rangle_{\text {jet }}^{(i)}\right],
\end{aligned}
$$

where $\langle R\rangle_{\text {jet }}^{(i)}$ is the jet acceleration's potential at the location of planet $(i)$. For consistency with the validity domain of the second-order planetary perturbation potential, we substitute $I$ for $\sin I$ in the expression (1). 
Introducing the vector $\mathbf{E}=\left(h_{1}, h_{2}, k_{1}, k_{2}, p_{1}, p_{2}, q_{1}, q_{2}\right)$, the equations of motion (3) take the simple form:

$$
\dot{\mathbf{E}}=L \cdot \mathbf{E}
$$

where the matrix $L$ is given as:

$$
L=\left[\begin{array}{cccccccc}
0 & 0 & G_{11} & G_{12} & v_{\mathrm{A}}^{(1)} & 0 & 0 & 0 \\
0 & 0 & G_{21} & G_{22} & 0 & v_{\mathrm{A}}^{(2)} & 0 & 0 \\
-G_{11} & -G_{12} & 0 & 0 & 0 & 0 & v_{\mathrm{A}}^{(1)} & 0 \\
-G_{21} & -G_{22} & 0 & 0 & 0 & 0 & 0 & v_{\mathrm{A}}^{(2)} \\
-v_{\mathrm{A}}^{(1)} & 0 & 0 & 0 & 0 & 0 & -G_{11} & G_{11} \\
0 & -v_{\mathrm{A}}^{(2)} & 0 & 0 & 0 & 0 & G_{22} & -G_{22} \\
0 & 0 & -v_{\mathrm{A}}^{(1)} & 0 & G_{11} & -G_{11} & 0 & 0 \\
0 & 0 & 0 & -v_{\mathrm{A}}^{(2)} & -G_{22} & G_{22} & 0 & 0
\end{array}\right]
$$

with:

$$
\begin{gathered}
G_{11}=\frac{n_{1}}{4} m_{2} \alpha^{2} b_{3 / 2}^{(1)}, \quad G_{22}=\frac{n_{1}}{4} m_{1} \alpha^{5 / 2} b_{3 / 2}^{(1)}, \\
G_{12}=-\frac{n_{1}}{4} m_{2} \alpha^{2} b_{3 / 2}^{(2)}, \quad G_{21}=-\frac{n_{1}}{4} m_{1} \alpha^{5 / 2} b_{3 / 2}^{(2)}, \\
v_{\mathrm{A}}^{(i)}=\frac{3 A}{2 n_{i} a_{i}} .
\end{gathered}
$$

To find the eccentricity and inclination expressions, we have to diagonalize the system (5) and use the initial orbital state of the two planets in terms of the vector $\mathbf{E}$ to identify its projections on the eigenvectors. As we are interested primarily in planetary systems on initially circular coplanar orbits, the acceleration's inclination $I_{0}$ with the planetary orbital plane implies:

$$
\mathbf{E}(t=0)=I_{0}(0,0,0,0,0,0,1,1) .
$$

Before using the solution of the secular system, we remark that: first, the addition of the acceleration term in the form of the new terms $v_{\mathrm{A}}^{(i)}$, lifts the degeneracy of the secular twobody problem. Indeed if $v_{\mathrm{A}}^{(i)}=0$ (i.e. there is no external acceleration), the matrix (5) can be split up into two separate blocs for the eccentricity and inclination leading, respectively, to two eigenmodes for the eccentricity and one eigenmode for the inclination. The latter is simply related to the arbitrary choice of a reference plane for the inclination. In contrast, the jet acceleration, the eccentricity and inclination variations are mixed. The second remark concerns the scaling of the eccentricity and inclination solution. Since the differential system representing the planets' eccentricities and inclinations is linear and since the initial state is proportional to $I_{0}$, the solutions for $e$ and $I$ will be proportional to $I_{0}$. This tells us that the solution is valid for any inclination provided that it is covered by the second-order approximation. As we shall see in the next section, the integration of the full equations of motion for an acceleration with $I_{0}=30^{\circ}$ shows that the agreement with the secular solution is reasonable. That is why we will not pay much attention to the actual amplitude of $I_{0}$ and quote mostly $e / I_{0}$ and $I / I_{0}$. This is consistent with the main goal of the paper as it was shown in N05 that a conservative constant-direction acceleration yields periodic oscillations of maximum amplitude $\sin I_{0}$ ( $I_{0}$ in the second-order approximation). Mutual perturbations cause the orbits to precess and tend to reduce the maximum eccentricity, it is therefore, natural to ascertain the effect of mutual planetary perturbations by scaling the eccentricity and inclination to $I_{0}$. 


\section{Comparison with the integration of the full equations of motion}

To illustrate the competition between mutual planetary perturbations and the eccentricity excitation by jet acceleration, N05 used a direct integration of the full equations of motion of the Jupiter-Saturn system. The set up was such that Jupiter and Saturn had circular coplanar orbits with their current semi-major axes. The inclination of the perturbing acceleration was $30^{\circ}$ and two different acceleration strengths, $A=2 \times 10^{-12}$ and $2 \times 10^{-14} \mathrm{~km} \mathrm{~s}^{-2}$ corresponding to the locations of the Keplerian boundary $a_{\mathrm{kplr}}$ at $10^{3}$ and $10^{4} \mathrm{AU}$, respectively. The Keplerian boundary corresponds to the location where the jet's acceleration balances the gravitational acceleration by the host star. For a stationary jet, objects with orbital semi-major axes beyond $a_{\mathrm{kplr}}$ are lost to the interstellar medium. In terms of $a_{\mathrm{kplr}}$, the acceleration is given as:

$$
\left|A_{0}\right| \simeq 2 \times 10^{-12}\left(\frac{M+m}{M_{\odot}}\right)\left(\frac{10^{3} \mathrm{AU}}{a_{\mathrm{kplr}}}\right)^{2} \mathrm{~km} \mathrm{~s}^{-2}
$$

and the corresponding excitation period, $T_{\mathrm{A}}=2 \pi / n_{\mathrm{A}}$, is given as:

$$
T_{\mathrm{A}} \simeq 10^{6}\left(\frac{M+m}{M_{\odot}}\right)^{1 / 2}\left(\frac{a_{\mathrm{kplr}}}{10^{3} \mathrm{AU}}\right)^{2}\left(\frac{1 \mathrm{AU}}{a}\right)^{1 / 2} \text { years }
$$

In Fig. 1, we compare the results of the numerical integrations using the full equations of motion with the solution of the secular systems (4)-(9) for an acceleration of Keplerian boundary
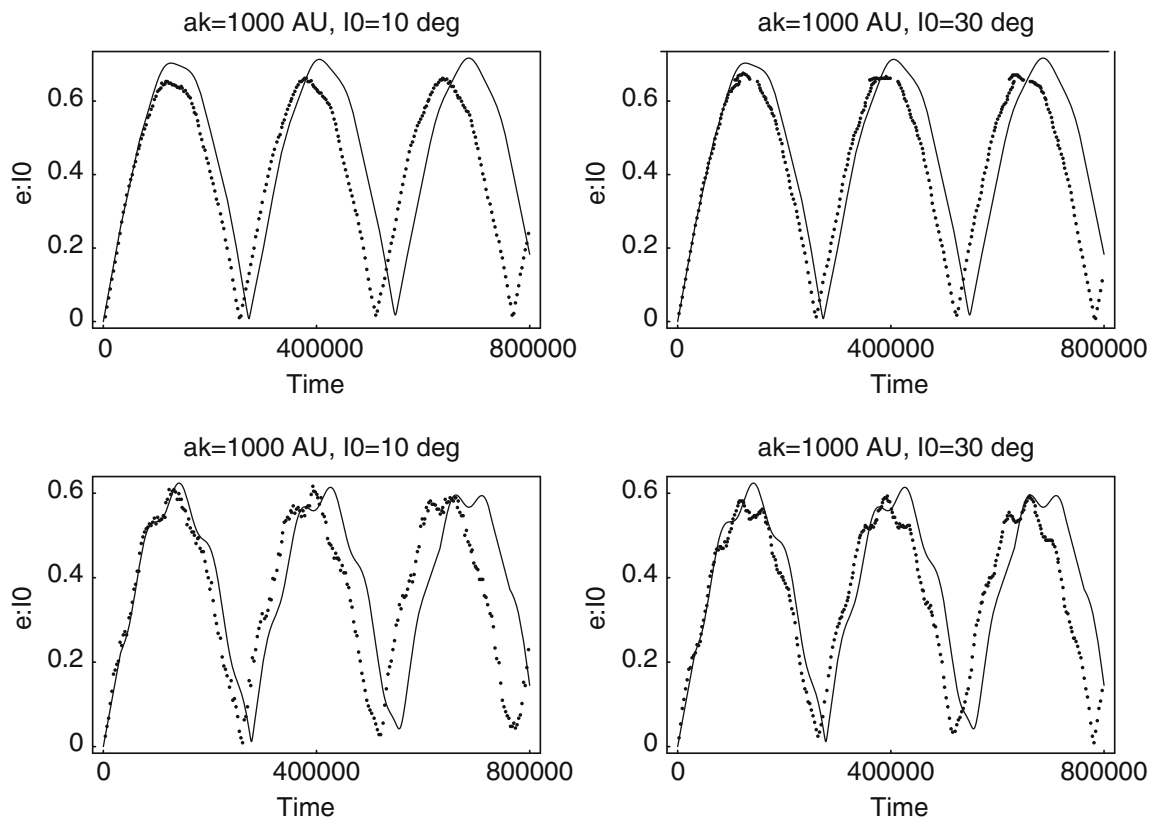

Fig. 1 The ratio of the eccentricity to the inclination as a function of time for Jupiter (upper panels) and Saturn (lower panels). The solid lines are obtained from the solution of the linear systems (4)-(9). The dotted lines are obtained from the direct integration of the full equations of motion, where Jupiter and Saturn have zero initial eccentricities. The two columns correspond to the two jet angles 10 and $30^{\circ}$. In all cases, the acceleration is $A=2 \times 10^{-12} \mathrm{~km} \mathrm{~s}^{-2}$ corresponding to $a_{\mathrm{kplr}}=1000 \mathrm{AU}$. Note that the dotted lines, we divided the eccentricity by $\sin I_{0}$ instead of $I_{0}$ for consistency with the full (non-linear) solution derived in N05 
$a_{\mathrm{kplr}}=1000 \mathrm{AU}$. As explained in the previous section, the ratio of the eccentricity $e$ to the jet inclination $I_{0}$ in the case of the secular system and to $\sin I_{0}$ for the numerical integrations is the relevant measure of excitation. We note three features: (1) the linear solution is a good approximation for relatively high eccentricities, $\sim 0.3$, and for relatively large inclinations, $\sim 30^{\circ}$. (2) The excitation period is somewhat overestimated for both small and large inclinations while (3) the amplitudes are somewhat underestimated for both inclinations. The agreement is only slightly better for smaller inclinations.

A stronger acceleration with a Keplerian boundary at $a_{\mathrm{kplr}}=500 \mathrm{AU}$ reveals similar features as seen in Fig. 2. Only in this case, the amplitudes of Jupiter are better reproduced than those with a weaker acceleration. However, this good fit is lost for larger times as it can be seen on Saturn's amplitudes.

The better agreement for Jupiter and the loss of accuracy with time suggest that the Jupiter-Saturn interaction combined to the jet acceleration is not purely secular in time, when observed over longer excitation cycles. This in turn suggests that mean motion resonances, which are neglected in the secular approach affect the eccentricity evolution over a time longer than the excitation time scale. In order to confirm this hypothesis, we study the previous Jupiter-Saturn configuration but use smaller masses for the two planets. Figure 3 shows the eccentricity excitation in such cases. The better agreement of the two approaches is already visible if we reduce Saturn's mass. An almost perfect agreement can be obtained if both planets_-Jupiter and Saturn-have smaller masses and the jet angle is small too.

We conclude that our method yields good results over the first excitation cycles when non-secular long-period perturbations do not have a pronounced effect on the eccentricity excitation. Such an agreement is sufficient for our purposes because as it has been shown in
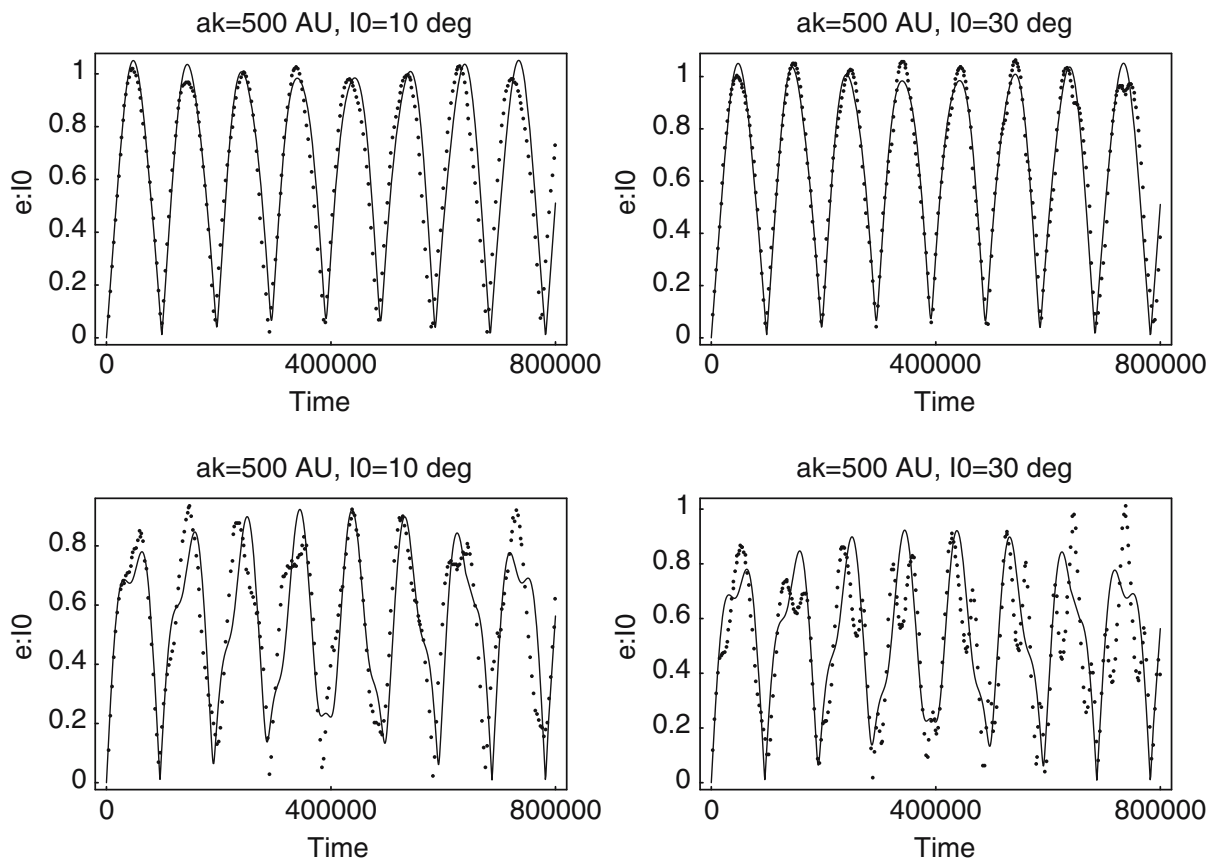

Fig. 2 Same as Fig. 1, but for a stronger acceleration $A=8 \times 10^{-12} \mathrm{~km} \mathrm{~s}^{-2}$ corresponding to $a_{\mathrm{kplr}}=500 \mathrm{AU}$. The upper panels correspond to Jupiter and the lower panels to Saturn 

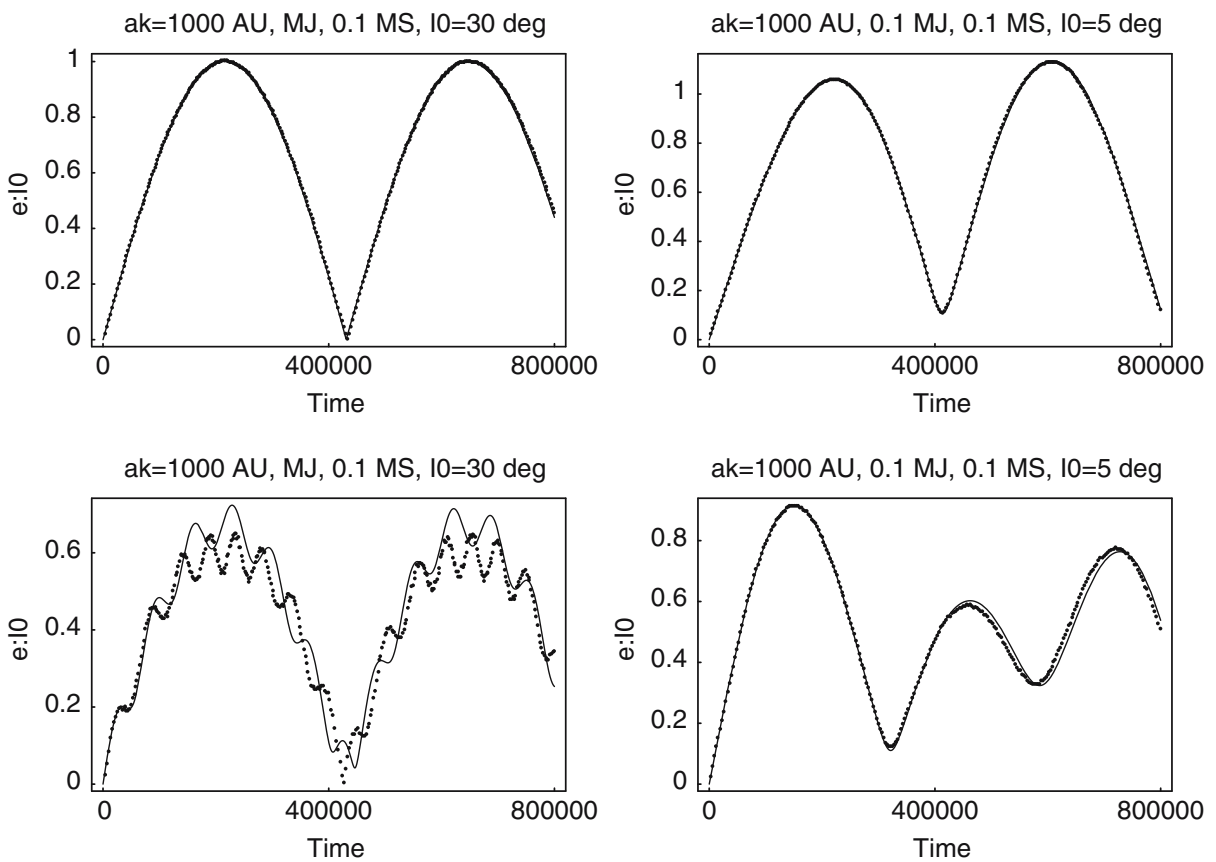

Fig. 3 The ratio of the eccentricity to the inclination as a function of time for two configurations. In the first configuration (left column), Saturn (lower plot) has a tenth of its actual mass; Jupiter's mass is set to its current value (upper plot). The jet angle is $30^{\circ}$ and the Keplerian boundary is at $a_{\mathrm{kplr}}=1000$ AU. In the second configuration (right columns), both Jupiter (upper plot) and Saturn (lower plot) have a tenth of their actual masses. The jet angle is chosen as $5^{\circ}$ to enhance the agreement with the direct numerical integrations; the Keplerian boundary is at $a_{\mathrm{kplr}}=1000 \mathrm{AU}$

N05 (1) the modeling of a realistic time dependent jet amounts to a simple convolution of the secular oscillations that we have described and the time window of the jet episode, and (2) typical action times for stellar jets are usually smaller than half the excitation time. The linear secular method is therefore, satisfactory to assess the influence of mutual planetary perturbations that are mainly secular over a single oscillation cycle.

\section{Effect of the acceleration's strength}

To characterize the relative importance of mutual gravitational perturbations and jet eccentricity excitation, we examine the dependence of the fundamental secular eigenmodes and the corresponding amplitudes as a function of the accelerations strength. For the acceleration strength, we take the more physical parameter $a_{\text {kplr }}$ that shows for instance where a putative disk is truncated by the jet's action. We also note that henceforth we shall not refer to the jet inclination because we normalize the eccentricity of both planets to $I_{0}$ (see Sect. 2). In Fig. 4, we illustrate the variation of the secular eigenperiods with $a_{\mathrm{kplr}}$. There are four eigenperiods, three of which tend to the free eigenmodes of the Jupiter-Saturn System: $T_{1}=375,000$ years, $T_{2}=60,000$ years, and $T_{3}=51,000$ years (N05, Murray and Dermott 1999). The last frequency $T_{4}$ is that of the jet excitation; it varies by two orders of magnitude as $a_{\mathrm{kplr}}$ varies from $\sim 10^{2}$ to $10^{3} \mathrm{AU}$ as roughly expected from expression (11). Writing the eccentricity vectors of the two planets as: 

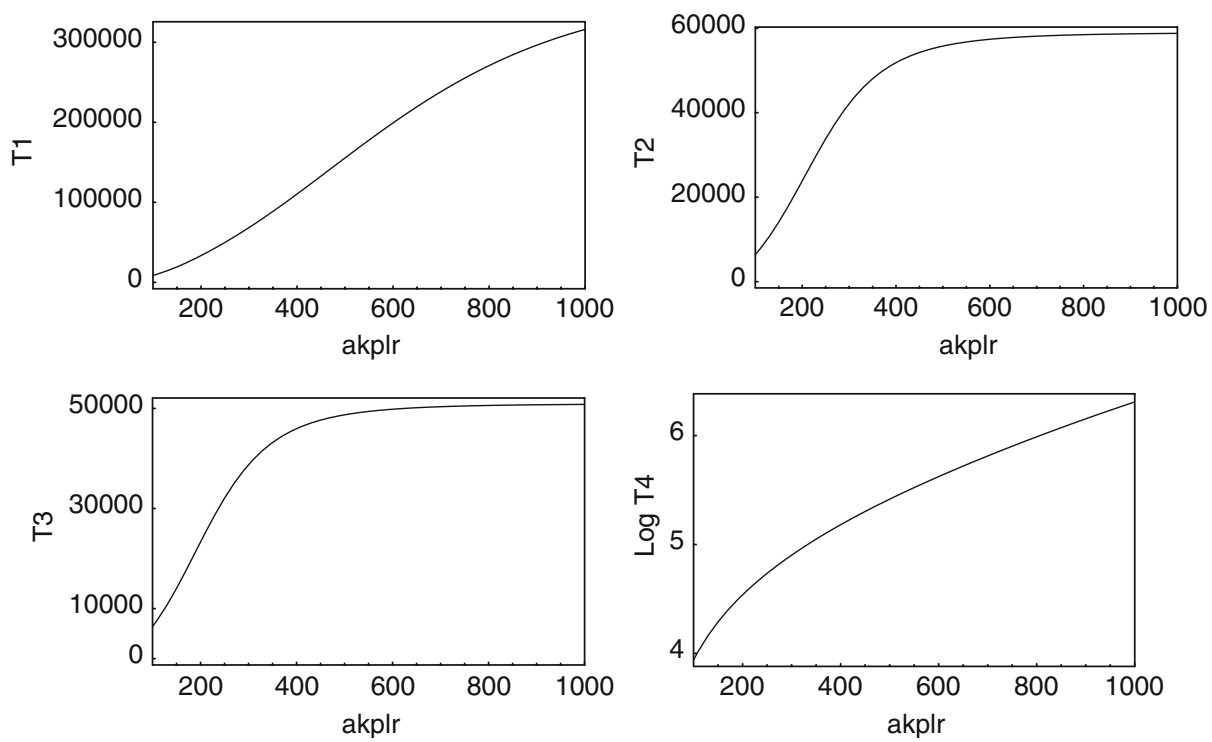

Fig. 4 Fundamental secular eigenperiods of the Jupiter-Saturn system as a function of the acceleration's strength represented by $a_{\mathrm{kplr}}(\mathrm{AU})$. The eigenperiods tend to the well known free periods as the acceleration gets weaker

$$
\mathbf{e}_{i}=I_{0} \sum_{k=1}^{4} \mathbf{e}_{i k},
$$

where $i=1$ for the inner planet (Jupiter) and 2 for the outer planet (Saturn) and the sum runs over the four eigenmodes, we show the amplitudes of the eccentricity vectors $e_{i k}$ for Jupiter in Fig. 5 and Saturn in Fig. 6. We note that the dominant eccentricity modes for Jupiter are 1 and 4 while all modes contribute to the eccentricity of Saturn.

\section{Effect of the planets' mass ratio}

We ascertain the effect of the planets' mass ratio by varying the mass of the JupiterSaturn system. We consider two cases: first, Saturn is left unchanged while Jupiter's mass is varied between 0.1 and $1 M_{\mathrm{J}}$; the semi-majors axes are unchanged. The acceleration strength corresponds to a Keplerian boundary at $a_{\mathrm{kplr}}=500 \mathrm{AU}$. The eigenmode amplitudes are shown in Fig. (7) for both the inner planet $\left(e_{1}\right)$ and Saturn $\left(e_{2}\right)$ as a function of their mass ratio. We observe the expected trend that the smaller the inner planet, the larger the excited eigenmodes. Fixing Jupiter's mass and varying Saturn's leads to the eigenmode amplitudes shown in Fig. 8. We note the degeneracy of the eigenmodes as the outer planet's mass decreases (mass ratio of inner to outer $\sim 35$ ). We remark however, that these eigenmodes should be used to obtain an estimate of the maximum eccentricity and do not exactly reproduce the eccentricity evolution beyond the first excitation cycle. This has already been encountered in Sect. 3, where we explained that the effect of mean motion resonances may cause departures from the purely secular evolution of the linear system (see also Fig. 2). 

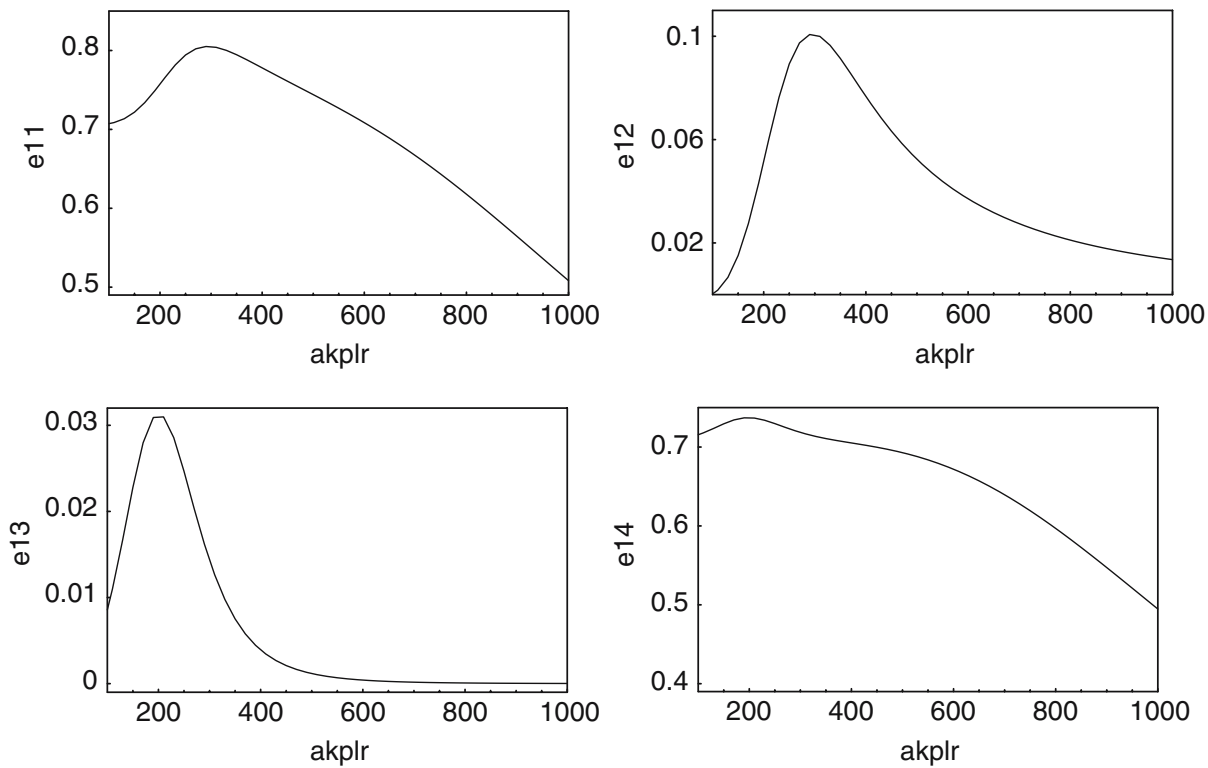

Fig. 5 Amplitudes of the secular eigenmodes of Jupiter as a function of the acceleration's strength represented by $a_{\mathrm{kplr}}(\mathrm{AU})$. Note that the amplitudes have been divided by $I_{0}$ (see Sect. 2)
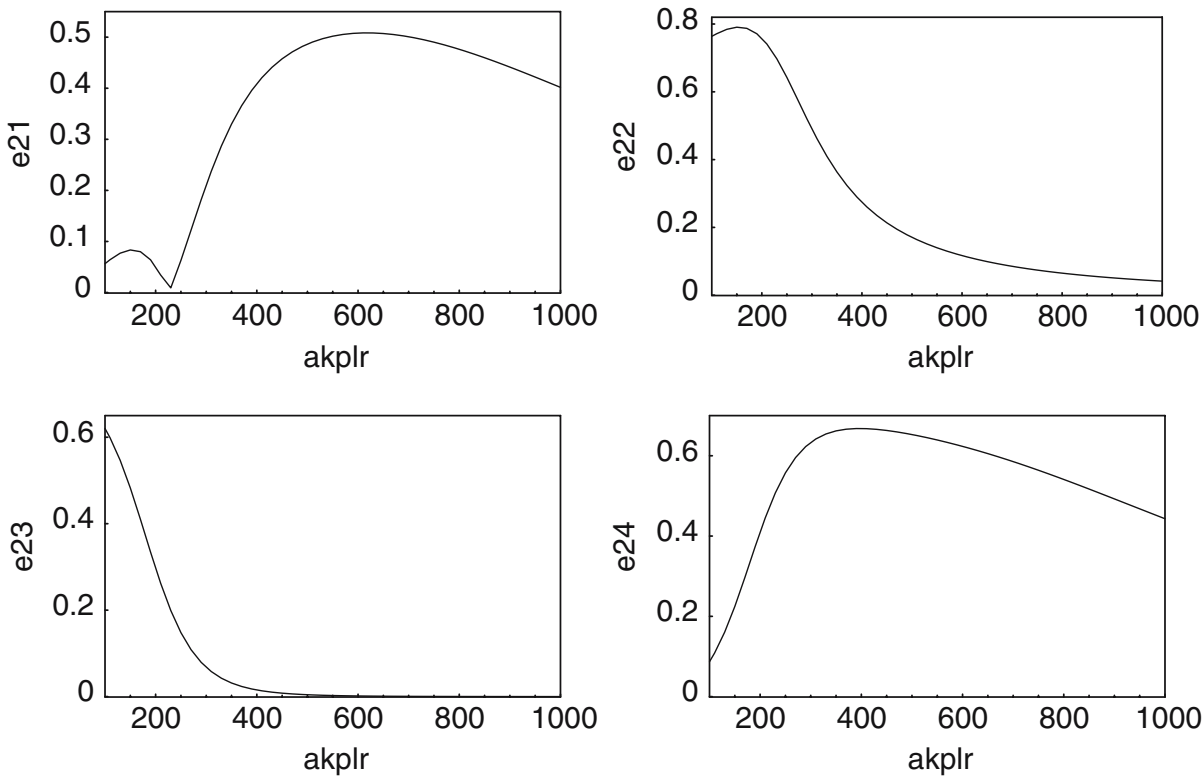

Fig. 6 Amplitudes of the secular eigenmodes of Saturn as a function of the acceleration's strength represented by $a_{\mathrm{kplr}}$ (AU). Note that the amplitudes have been divided by $I_{0}$ (see Sect. 2) 

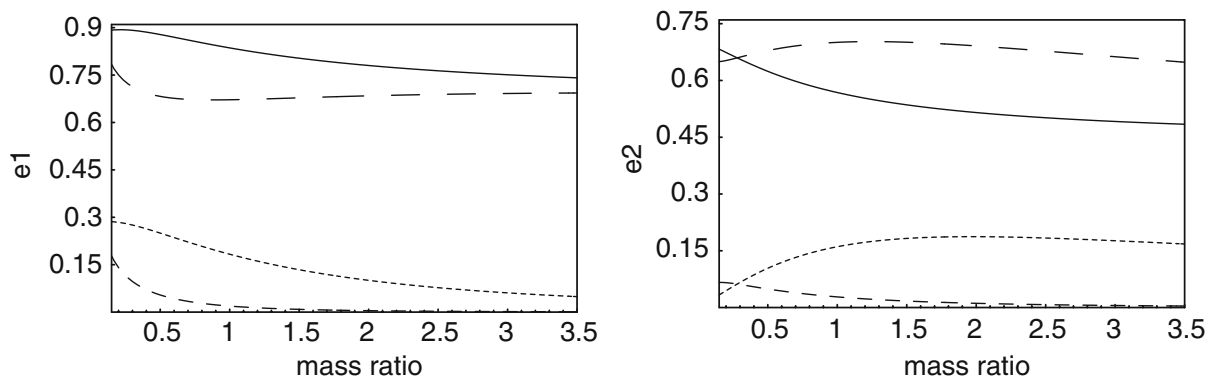

Fig. 7 Amplitudes of the secular eigenmodes of Saturn (right panel) interacting with an inner planet (left panel) at Jupiter's semi-major axis whose mass varies from 0.1 to $1 M_{\mathrm{J}}$. The horizontal axis shows the mass ratio of the inner planet to the outer planet. The amplitudes correspond to the eigenmodes 1 (solid), 2 (shortdashed), 3 (medium-dashed) and 4 (long-dashed)
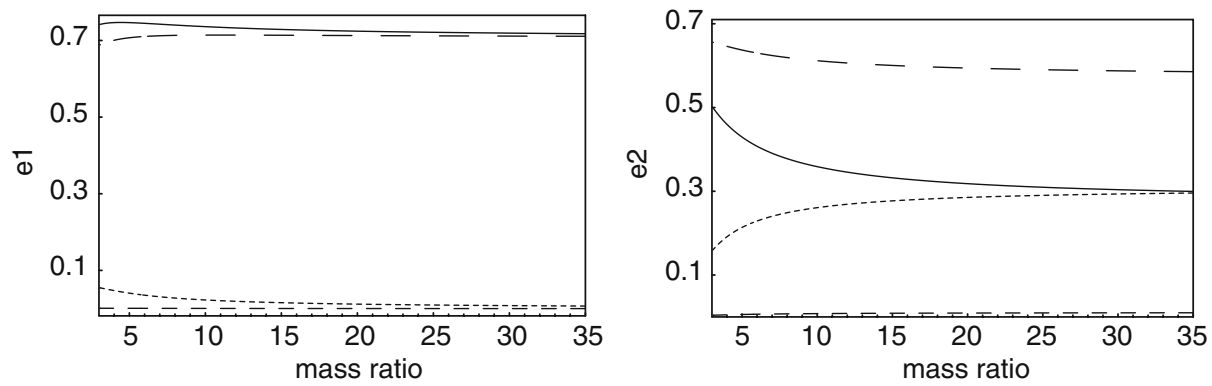

Fig. 8 Amplitudes of the secular eigenmodes of Jupiter (left panel) interacting with an outer planet (right panel) at Saturn's semi-major axis whose mass varies from 0.1 to $1 M_{\mathrm{S}}$. The horizontal axis shows the mass ratio of the inner planet to the outer planet. The amplitudes correspond to the eigenmodes 1 (solid), 2 (shortdashed), 3 (medium-dashed), and 4 (long-dashed)

\section{Effect of the planets' relative distance}

The effect of mutual interactions is diminished by an increasing distance between the two planets. Equivalently, one can choose a weaker acceleration that has a smaller Keplerian boundary radius, $a_{\mathrm{kplr}}$. This is recovered in the linear model as it is shown in Fig. 9 for an acceleration with a Keplerian boundary at 500 AU. In this example, Saturn's semi-major axis was kept at its current value while Jupiter's semi-major axis was varied in the range set by the 2:1 and the 4:1 resonances. We note a curious mode amplitude exchange for Saturn at $\alpha=0.475$. An important conclusion that we can draw from the previous two sections is that an acceleration with a Keplerian boundary at $500 \mathrm{AU}$ while displaying the correct trends as the mass ratio or the semi-major axis vary, is relatively strong so as to excite the two planets' eccentricities. Excitation does actually occur only if the direction of acceleration represented by the factor $I_{0}$ (or more precisely $\sin I_{0}$ ), that appears in the eccentricity expression (12), is not too small.

\section{Application to HD 108874, 47 Uma, and HD 12661}

To illustrate the use of the secular method in the case of extrasolar planets, we determine the eigenmode amplitudes as a function of the acceleration's strength represented by the radius 

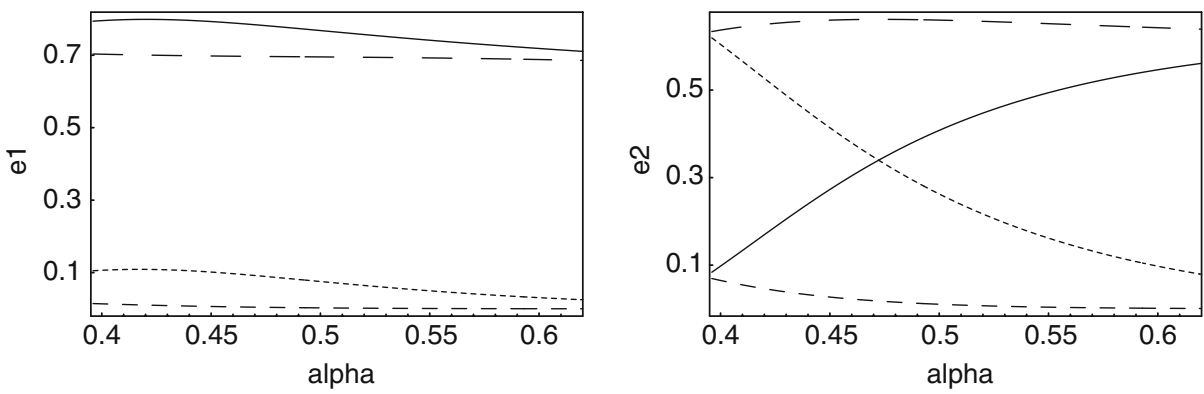

Fig. 9 Amplitudes of the secular eigenmodes of Jupiter (left panel) and Saturn (right panel) as a function of the semi-major axis ratio. Saturn's semi-major axis is set at its current value 9.2525 and Jupiter's varies. The amplitudes correspond to the eigenmodes 1 (solid), 2 (short-dashed), 3 (medium-dashed), and 4 (long-dashed)

of the Keplerian boundary for the two-planet systems of HD108874 (Vogt et al. 2005), 47 Uma (Fischer et al. 2002a) and HD 12661 (Fischer et al. 2002b). The corresponding orbital data are given in Table 1 (Appendix) and the amplitudes in Fig. 10.

Assuming a reference inclination of $I_{0}=20^{\circ}\left(\sin I_{0}=0.34\right)$, our excitation criterion for these systems is that the maximum value of all eigenmode amplitudes be larger than the observed eccentricity values. Such a criterion is not the most conservative nor is it the most relaxed one. The latter comes from the fact that the eccentricity of each planet is the sum (with the corresponding phases) of the various modes and is always likely to be larger than the largest mode amplitude.

For the chosen criterion, the HD 108874 planets are different, while the inner planet requires only a small eccentricity and therefore satisfies the criterion quite well, HD 108874c with an eccentricity of 0.25 barely meets the criterion for a strong acceleration of Keplerian boundary $a_{\mathrm{kplr}}=150 \mathrm{AU}$. The planets of 47 Uma require stronger accelerations because of their larger masses (and hence stronger gravitational interaction), while those of HD 12661 with their low-eccentricity values satisfy the criterion for $a_{\mathrm{kplr}} \leq 350 \mathrm{AU}$. In view of this analysis, it is important to bear in mind that we have assumed a constant-direction acceleration throughout this paper, which in reality represents slowly-precessing jets only. The fact that the planets of 47 Uma, for instance, do not meet the excitation criterion does not imply that the jet acceleration model is not able to explain the planets' orbital configuration. It has been shown in N05 that the resonance between the jet's precession frequency and the excitation frequency $n_{\mathrm{A}}$ is able to excite the eccentricities to unity even for nearly perpendicular jets. In this instance, it is interesting to note that HD $108874 \mathrm{~b}$ has a small eccentricity indicating the possibility of eccentricity damping that is characteristic of the orbital region inside the resonance radius. This suggests that the jet resonance is located outside $1 \mathrm{AU}$ and that the more eccentric outer planet lies in the resonance region.

\section{Summary}

We have presented a simple and fast method to determine the effect of a jet induced acceleration on a two-planet system by applying linear secular perturbation theory. We combined the Laplace-Lagrange theory of first-order in the masses and second-order in the eccentricities secular potential with the secular potential of a jet acceleration derived in N05. This has enabled us (1) to verify the general excitation trends as the acceleration's strength, the planetary mass ratio and the relative semi-major axes vary and (2) to estimate of the accelera- 

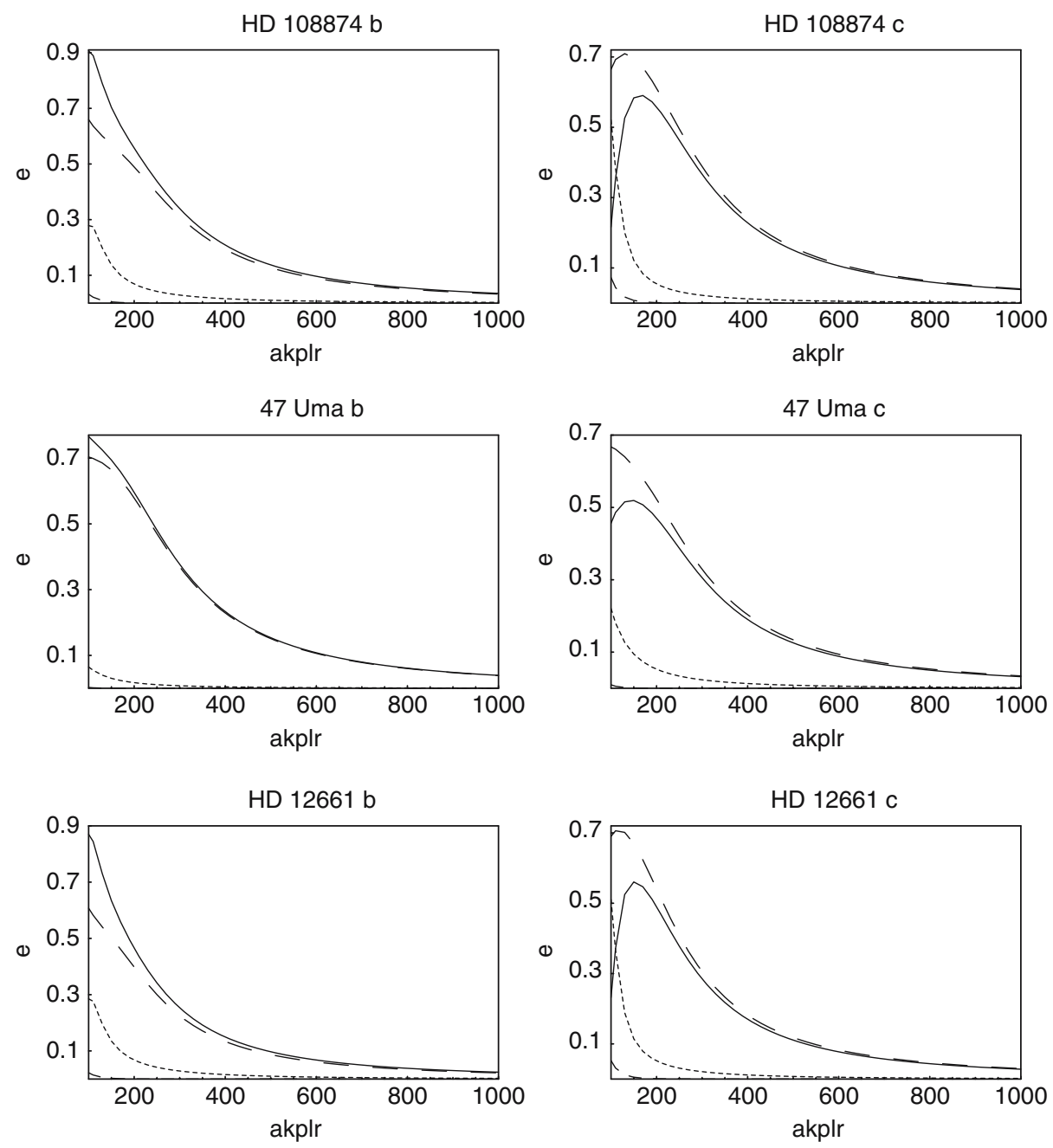

Fig. 10 Amplitudes of the secular eigenmodes of the companions to HD 108874, 47 Uma, and HD 12661 as a function of the radius of the Keplerian boundary. The amplitudes correspond to the eigenmodes 1 (solid), 2 (short-dashed), 3 (medium-dashed), and 4 (long-dashed)

tion strengths required in the extrasolar systems of HD 108874, 47 Uma, and HD 12661. We found that the first two systems were likely excited by the resonance of the jet's precession frequency with its natural excitation frequency that is known to yield maximum eccentricity values near unity regardless of the jet inclination angle. The eccentricities of the planets around HD 12661 can be explained by the acceleration of a slowly precessing jet modeled with the linear theory. Extensions of this work include generalizing the linear secular analysis to any number of planets, and also the coupling of the secular dynamics to specific mean motion resonances. The treatment of the jet resonance however, can not be dealt with in the linear regime and requires the Kozai potential instead of that in Eq. (2).

Acknowledgements The authors thank the referee for helpful comments. FN thanks Alessandra Celletti and the Celmec IV organizing committee for their invitation and the Programme National de Planétologie for partial financial support. JLZ was supported by the Natural Science Foundation of China (Grant 10233020) 
and the program for New Century Excellent Talents in Universities of China (Grant 04-0468). Part of this work was done while JLZ was on a CNRS-NSFC exchange programme in the Observatoire de la Côte d'Azur; he thanks Christiane Froeschlé, Elena Lega, Claude Froeschlé and the Cassiopée Department for their hospitality.

\section{Appendix}

Table 1 Orbital elements and masses of the companions to HD 108874, 47 Uma, and HD 12661 (Fischer et al. 2002a, b; Vogt et al. 2005)

\begin{tabular}{lcccc}
\hline Companion & $m / M_{\mathrm{J}} \sin i$ & $a$ & $e$ & $M_{*} / M_{\odot}$ \\
\hline HD 108874b & 1.36 & 1.051 & 0.07 & 1 \\
HD 108874c & 1.018 & 2.68 & 0.25 & 1 \\
47 Uma b & 2.54 & 2.09 & 0.35 & 1.03 \\
47 Uma c & 0.76 & 3.73 & 0.2 & 1.03 \\
HD 12661b & 2.3 & 0.83 & 0.06 & 1.07 \\
HD 12661c & 1.57 & 2.56 & 0.1 & 1.07 \\
\hline
\end{tabular}

\section{References}

Chiang, E.I., Fischer, D., Thommes, E.: Excitation of orbital eccentricities of extrasolar planets by repeated resonance crossings. Astrophys. J. 564, L105-L109 (2002)

Fischer, D.A., Marcy, G.W., Butler, R.P., Laughlin, G., Vogt, S.S.: A second planet orbiting 47 ursae majoris. Astrophys. J. 564, 1028-1034 (2002a)

Fischer, D.A., Marcy, G.W., Butler, R.P., Vogt, S.S., Henry, G.W., Pourbaix, D., Walp, B., Misch, A.A., Wirght, J.T.: A planetary companion to HD 40979 and additional planets orbiting HD 12661 and HD 385291. Astrophys. J. 586, 1394-1408 (2002b)

Ford, E.B, Rasio, F.A., Yu, K.: Dynamical instabilities in extrasolar planetary systems. In: Deming, D. Seager S. (eds.) Scientific Frontiers in Research on Extrasolar Planets ASP Conf. Series 294, 181-188 (2003)

Goldreich, P., Tremaine, S.: The origin of the eccentricities of the rings of uranus. Astrophys. J. 243, $1062-1075$ (1981)

Holman, M., Touma, J., Tremaine, S.: Chaotic variations in the eccentricity of the planet orbiting 16 Cyg B. Nature 386, 254-256 (1997)

Lee, M.H., Peale S.J.: Dynamics and origin of the 2:1 orbital resonances of the GJ 876 planets. Astrophys. J. 567, 596-609 (2002)

Marzari, F., Weidenschilling, S.J.: Eccentric extrasolar planets: the jumping Jupiter model. Icarus 156, 570-579 (2002)

Murray, C.D., Dermott, S.F.: Solar system dynamics. Cambridge University Press, Cambridge (1999)

Namouni, F.: On the origin of the eccentricities of extrasolar planets. Astron. J. 130, 280-294 (2005)

Papaloizou, J.C.B., Terquem, C.: Dynamical relaxation and massive extrasolar planets. MNRAS 325, 221-230 (2001)

Stepinski, T.F., Black, D.C.: On orbital elements of extrasolar planetary candidates and spectroscopic binaries. Astron. Astrophys. 371, 250-259 (2001)

Takeda, G., Rasio, F.: High orbital eccentricities of extrasolar planets induced by the Kozai mechanism. Astrophys. J, 512, 1001-1010 (2005)

Terquem, C., Papaloizou, J.C.B.: Dynamical relaxation and the orbits of low-mass extrasolar planets. MNRAS 332, L39-L43 (2002)

Tremaine, S., Zakamska, N.: Extrasolar planet orbits and eccentricities. In: Holt, S.S. Deming, D. (eds.) The Search for Other Worlds, AIP Conf. Proc. 713, 243-252 (2004)

Vogt, S.S., Butler, R.P., Marcy, G.W., Fischer, D.A., Henry, G.W., Laughlin, G., Wright, J.T., Johnson, J.A.: Five new multicomponent planetary systems. Astrophys. J. 632, 638-658 (2005)

Zakamska, N., Tremaine, S.: Excitation and propagation of eccentricity disturbances in planetary systems. Astron. J. 128, 869-877 (2004) 